\title{
Novel Synthesis of Polymers with Well-Defined Structures Based on Selective Reaction of Cyclic Sulfides and Cyclic Ethers Catalyzed by Quaternary Onium Salts
}

\author{
By Atsushi KAMEYAMA ${ }^{1, *}$ and Tadatomi NISHIKUBO ${ }^{2}$
}

Novel syntheses of polymers with well-defined structures were developed on the basis of the selective reactions of thiiranes and oxiranes with carboxylic esters or active chlorides using quaternary onium salts. (1) A new type of reactive polymers with pendant chloromethyl groups was synthesized by polyaddition of difunctional thiiranes or oxiranes with various organic dichlorides. (2) Polysulfides with controlled molecular weight and narrow molecular weight distribution were synthesized by ring-opening polymerization of thiiranes with carboxylic acid derivatives as initiators and quaternary onium salts as catalysts, in which acyl-groups transfer to the propagating center in each propagation step. The ring-opening polymerization of thiiranes has an advantage in the synthesis of specific shaped polymers such as star polymers and cyclic polymers. (3) Novel A-B type sequence-ordered copolymers were synthesized by alternating ring-opening polymerization of oxiranes with $\gamma$ thiobutyrolactone, or of oxetane with cyclic carboxylic anhydrides. (4) Sequence-ordered polymers were successfully synthesized on the basis of a new concept- "transformation of polymer backbones" using insertion of thiiranes and oxiranes into poly( $S$-thioester)s in the aforementioned reaction systems. The reaction mechanism and advantages of the reaction systems are also discussed.

KEY WORDS: Ring-opening Reaction / Thiirane / Oxirane / Quaternary Onium Salts / Acyl Transfer / Sequence-ordered Polymers /

Addition reactions of cyclic compounds such as cyclic ethers with certain reagents are useful synthetic methods for preparing polymeric materials. The addition reactions of oxiranes (epoxy) compounds have been used for syntheses of functional polymers from reactive polymers containing pendant oxirane groups. ${ }^{1}$ Epoxy resins with multiple oxirane groups are widely utilized with certain curing reagents to obtain thermal cured materials. ${ }^{2}$ These polymer syntheses are based on the addition reaction of oxiranes with such reagents as amines, carboxylic acids, etc. In general, the reactions provide mixtures of $\alpha$-addition and $\beta$-addition products (mixtures of regio-isomers). ${ }^{3}$ Regioselective ring-opening reactions of oxiranes are important research subjects in synthetic organic chemistry, ${ }^{4}$ but it has been thought that the reactions of oxiranes with the reagents mentioned are not suitable for precise syntheses of polymers with well-defined structures.

Recently, we have developed synthetic methods for functional polymers and chemical modification of polymers based on the regioselective ring-opening reactions of oxiranes with carboxylic esters catalyzed by quaternary onium salts. ${ }^{5}$ In a series of studies on ring-opening reaction of cyclic compounds with several reagents; we found novel regioselective reactions of thiiranes or oxiranes to be useful for syntheses of polymers with well-defined structures.

A significant advantage of the addition reactions of thiiranes or oxiranes with the reagents is the selective formation of $\beta$ addition products without ring-opening polymerization under neutral conditions, as shown in Scheme 1. Another interesting feature of the reactions is that the resulting molecules bear two types of functional groups. The reactions could be applied to controlled chain-growth polymerization of thiiranes, and step-growth polymerization of bi-functional cyclic thiiranes or cyclic ethers. We found reactions of thiiranes and oxiranes to be useful for the transformation of $\operatorname{poly}(S$-thioester $)$ s to obtain a new type of sequence-ordered polymers. We also found several ring-opening alternating polymerizations of the cyclic ethers that gave A-B type sequence-ordered polymers.

This review describes successful syntheses of polymers with well-defined structures on the basis of a novel reaction system; the selective reactions of thiiranes and oxiranes under neutral conditions.

\section{REGIOSELECTIVE RING-OPENING REACTIONS OF THIIRANES}

Cyclic sulfides and cyclic ethers are useful starting materials for preparing sulfur-containing polymeric materials. Thiiranes and thietanes are highly reactive cyclic sulfides with strain energies of $83 \mathrm{kcal} / \mathrm{mol}$ and $79 \mathrm{kcal} / \mathrm{mol}$, respectively. ${ }^{6} \mathrm{Sev}$ eral studies on cycloaddition of thiiranes with some reagents have been reported. Cycloaddition reaction of thiiranes with

\footnotetext{
${ }^{1}$ Department of Chemistry, Faculty of Engineering, Kanagawa University, Rokkakubashi, Kanagawa-ku, Yokohama 221-8686, Japan

${ }^{2}$ Department of Materials \& Life Chemistry, Faculty of Engineering, Kanagawa University, Rokkakubashi, Kanagawa-ku, Yokohama 221-8686, Japan

*To whom correspondence should be addressed (Tel: +81-45-481-5661, Fax: +81-45-413-9770, E-mail: kameya01@ kanagawa-u.ac.jp).
} 
Regioselective ring-opening reaction of cyclic sulfides and ethers

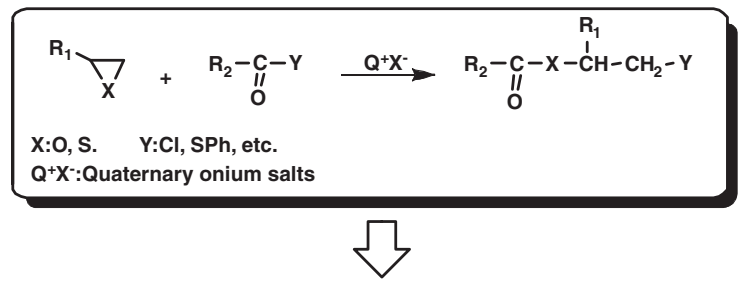

*Polymerization forming pendant reactive groups

*Acyl-transfer polymerization

*Transformation of polymer backbones

Scheme 1.

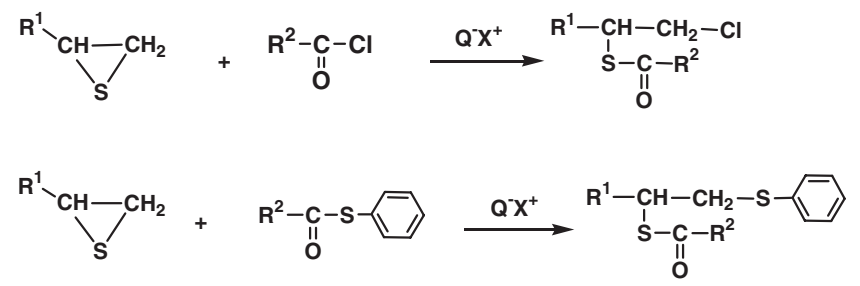

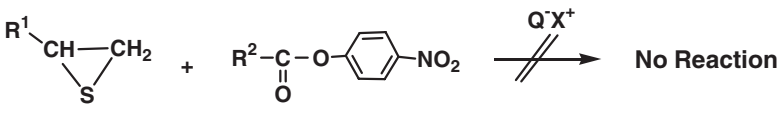

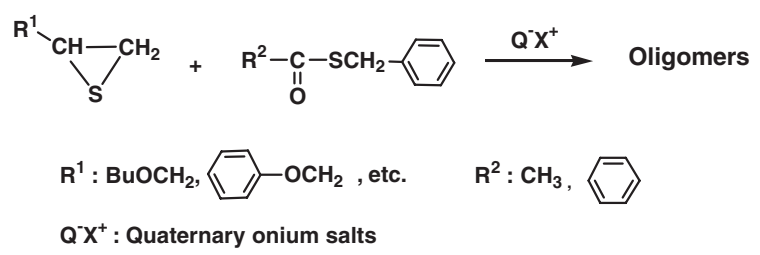

Scheme 2.

various reagents have been reported. Cycloaddition reactions of thiiranes with alkyl or aryl nitriles ${ }^{7}$ in the presence of strong acid, yielding thiazoline derivatives, have been studied. Cycloaddition reactions of thiiranes with carbon disulfide, ${ }^{8}$ ethyl- $\alpha$ cyanoacetate, ${ }^{9}$ thiocyanates, ${ }^{10}$ and with diethyl malonate, ${ }^{11}$ using various bases have also been reported. Surprisingly, textbooks ${ }^{12}$ and review articles ${ }^{13,14}$ on organic sulfur chemistry explain regioselective ring-opening reactions of thiiranes without commenting in detail. Except for ring-opening polymerization of thiiranes, ${ }^{15,16}$ a few studies on the ring-opening reactions of thiiranes with certain reagents have been reported. One case is the reaction of thiiranes with hydrogen chloride. ${ }^{17}$ Funahashi also attempted a ring-opening reaction of a thiirane with phenyl acetate ${ }^{18}$ using a base; however, the reaction was not successful. A ring-opening reaction of a thiirane with acetyl chloride catalyzed by cobalt(II) chloride was reported, however, the regioselectivity of the used thiirane was not sufficient. ${ }^{19}$

With this background, we studied regioselective ring-opening reactions of thiiranes with carboxylic acid derivatives (Scheme 2). ${ }^{20}$ As a typical example, the reaction of 3- butoxypropylene sulfide (BPS) with benzoyl chloride (BC) proceeded efficiently with suitable catalysts such as tetrabutylammonium bromide (TBAB), chloride (TBAC) and tetrabutylphosphonium chloride (TBPC) to give a product in almost quantitative yield. The ${ }^{1} \mathrm{H}$ NMR spectrum of the product proved that the reaction of BPS with BC proceeded with $100 \%$ regioselectivity to provide only the $\beta$-addition product. The reaction of other thiiranes with certain acyl chlorides also proceeded easily to give the corresponding $\beta$-addition products in high yield.

Although the reaction of BPS with $S$-phenyl thioacetate (PTA) did not occur in the absence of a catalyst, even at $90{ }^{\circ} \mathrm{C}$ for $1 \mathrm{~h}$, the reaction proceeded very efficiently with TBAC catalysts under the same conditions to form a $\beta$-addition product. In the reaction of BPS with $S$-benzyl thioacetate (BZTA), an $S$-alkyl thioester, the reaction product was not just an addition product but oligomers with number-average molecular weight $\left(M_{\mathrm{n}}\right)$ of 1500 (based on PS standards). The IR spectrum of the oligomer showed a weak peak due to $v(\mathrm{C}=\mathrm{O})$ of the $S$-thioester at $1690 \mathrm{~cm}^{-1}$. This result is understandable as follows: the reaction of BPS with BZTA produces the corresponding product, an $S$-alkyl thioester, in the initial stage. It seems that the reactivity of the resulting alkyl thioester group toward BPS is ordinarily the same as that of BZTA. Thus, the reaction of the $S$-alkyl thioester with BPS occurred in chain reaction mode to produce oligomers with terminal $S$-thioester groups.

On the other hand, when reactions of BPS with $O$-active esters, $p$-nitrophenyl acetate (NPA) or $p$-chlorophenyl acetate (CPA), were attempted using TBAC, the reaction did not occur under any conditions. The selectivity in the reactions of thiiranes with carboxylic acid derivatives can be understood considering the ability of the leaving groups of the carboxylic acid derivatives, as shown in Scheme 3. A counter-anion of quaternary onium halide $\left(\mathrm{Q}^{+} \mathrm{X}^{-}\right)$attacks the $\beta$-carbon of the thiirane to form an alkyl thiolate $\left(\mathrm{R}-\mathrm{S}^{-}\right)$, Intermediate I. Intermediate I reacts with carboxylic acid derivatives to form intermediate II. When the leaving ability of the leaving group (L) is greater than that of alkyl thiolate, $\mathrm{L}$ is released to replace a halide $\mathrm{X}^{-}$and recover $\mathrm{Q}^{+} \mathrm{X}^{-}$. The high regioselectivity of the reactions is attributable to steric hindrance between the substituent group of thiirane and the quaternary onium salts used. In the case of the reaction with $O$-aryl esters, the intermediate II would revert to the starting materials due to the equilibrium. When thiiranes react with $S$-alkyl thioesters, reactions of the $S$-alkyl thioester produced with the thiirane proceed in chain-reaction mode to produce oligomers.

In addition to the high regioselectivity of the reactions of thiiranes, another advantage of the reactions is the selective transformation of thiiranes under neutral conditions, because quaternary onium salts do not induce ring-opening polymerization of thiiranes, which are easily polymerized by acid or base catalysts. These regioselective reactions of thiiranes have high potential for the synthesis of sulfur-containing materials with well-defined structures. 


\section{Leaving ability : $\mathrm{Cl}^{-}>\mathrm{PhS}^{-}>\mathrm{PhCH}_{2} \mathrm{~S}^{-}>\mathrm{PhO}^{-}$}

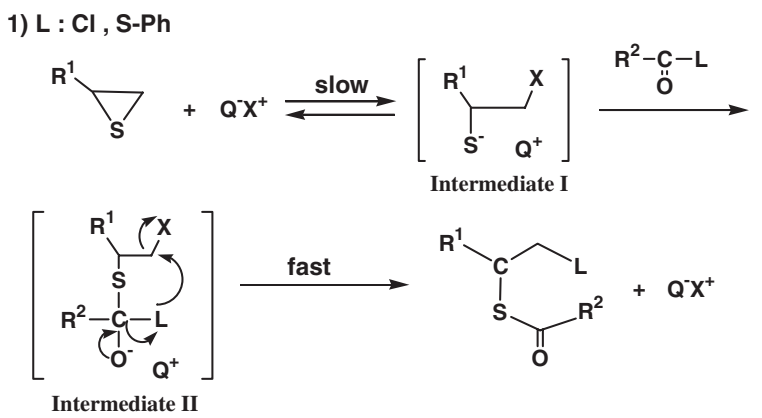

2) $\mathrm{L}: \mathrm{OPh}\left(p-\mathrm{NO}_{2}\right)$

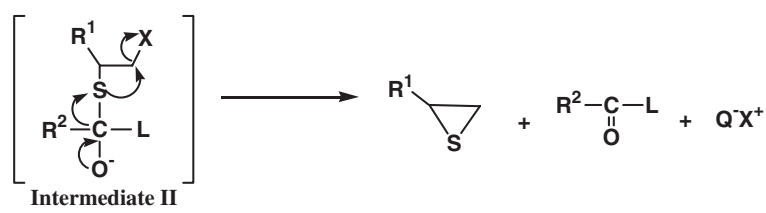

3) $\mathrm{L}: \mathrm{SCH}_{2} \mathrm{Ph}$

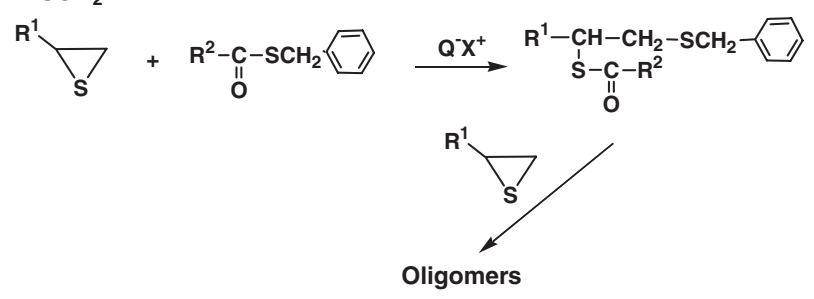

Scheme 3.

\section{SYNTHESIS OF REACTIVE POLYMERS WITH PENDANT CHLOROMETHYL GROUPS}

Poly(S-thioester)s are useful materials that have been prepared by the condensation ${ }^{21}$ of dithiols with dicarboxylic acid chlorides or diphenyl dicarboxylates, or by the ringopening polymerization of $\beta$-thiopropiolactones, ${ }^{22} \delta$-thiovalerolactones, ${ }^{23}$ or $\varepsilon$-thiocaprolactones ${ }^{24}$ using strong bases.

We developed a successful synthesis of poly ( $S$-thioester)s based on the addition reactions of thiiranes with carboxylic acid derivatives (Scheme 4). ${ }^{25} \mathrm{~A}$ unique point of the polymer synthesis is the simultaneous formation of $S$-thioester groups in the chain backbone and pendant chloromethyl groups. The polyaddition of bisphenol A di(2,3-epithiopropyl)ether (BPTE) with terephthaloyl chloride (TPC) using quaternary ammonium or phosphonium salts such as TBAC, TBAB, tetrabutylammonium iodide (TBAI) or tetrabutylphosphonium bromide (TBPB) proceeded smoothly to provide polymers with $M_{\mathrm{n}}$ of 20,000-25,000. As to the counter-anions of tetrabutylammonium halides, TBAC provided polymers with higher molecular weight, as compared with TBAB and TBAI. The polymer with the highest $M_{\mathrm{n}}$ of 30,000 was obtained from the reaction with TBPC under the same reaction conditions. This means that the catalysts with chloride as the counter anion have higher catalytic activity than those with bromide or iodide and suggests that the properties of the halides of quaternary onium salts such as the nucleophilicity and their leaving ability are very important in the reactions of thiiranes with carboxylic acid derivatives. ${ }^{20}$ It is also suggested that catalytic activity of the quaternary onium salts is affected by the nature of quaternary onium cations such as hydrophobicity and bulkiness due to their substituents. The reaction of BPTE with TPC in toluene at $90{ }^{\circ} \mathrm{C}$ was efficiently enhanced by the concentration of TBPC as the catalyst. The molecular weight of the polymer obtained increased from 30,000 to 50,000 when the concentration of TBPC was increased from $2 \mathrm{~mol} \%$ to $11 \mathrm{~mol} \%$. According to the mechanism of the reaction of thiiranes with carboxylic acid derivatives in Scheme 2, the polymerization rate depends on the concentration of the intermediate formed from thiiranes and quaternary onium salts. Various types of poly( $S$-thioester)s were synthesized by the polyaddition of BPTE with several diacyl chlorides such as adipoyl chloride (APC) and sebacoyl chloride (SCC). The poly( $S$-thioester)s obtained had high molecular weights, as high as 20,000-40,000, and were well characterized to show that the polymers had pendent chloromethyl groups formed by selective $\beta$-addition of thiirane groups. It is concluded that the polyaddition of bis(thiirane)s with diacyl chlorides using quaternary onium salts is a useful synthetic method for $\operatorname{poly}(S$-thioester $)$ s.

In a series of studies on novel ring-opening reactions of cyclic sulfides and cyclic ethers, we also succeeded in synthesizing novel reactive polymers with pendant chloromethyl groups based on the regioselective addition reactions of

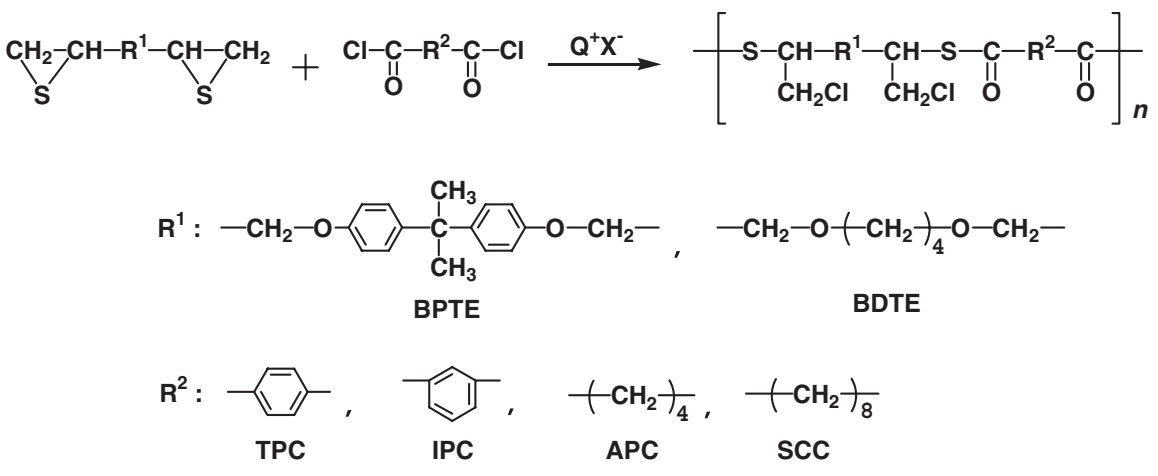

Scheme 4. 

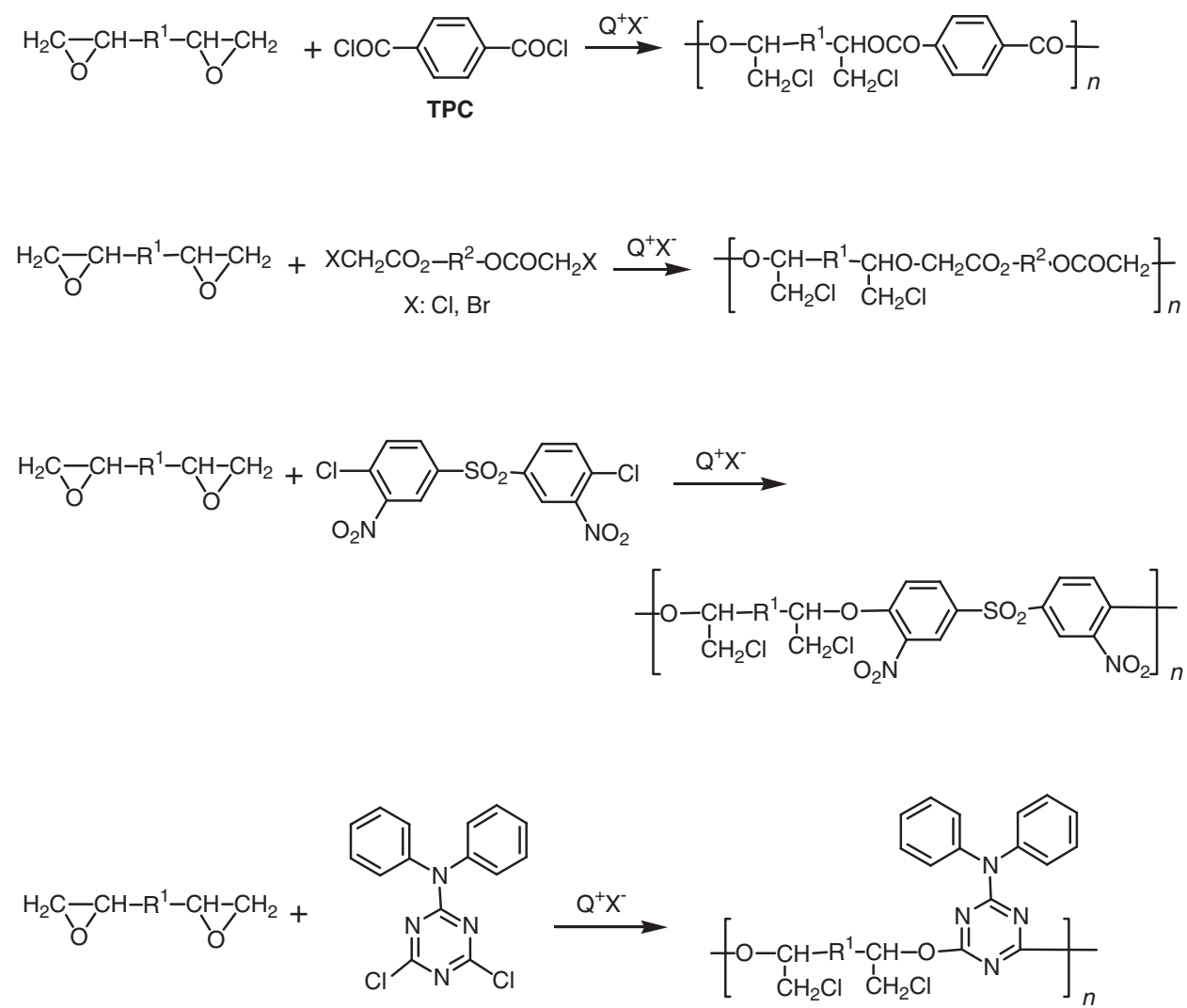

Scheme 5.

bis(epoxide)s with active organic dichlorides catalyzed by quaternary onium salts (Scheme 5). The regioselective polyaddition of bis(epoxide)s with diacyl chlorides provided poly(ester)s with pendant chloromethyl groups, and high molecular weights. ${ }^{26}$ The regioselective polyaddition of bis(epoxide)s with active aromatic dichlorides also provided reactive poly(ether)s with pendant chloromethyl groups. ${ }^{27}$ The polyaddition of bis(epoxide)s with bis(chloroacetoxy)esters or bis(bromoacetoxy)esters proceeded regioselectively to give poly(ether)s with pendant halomethyl groups. ${ }^{28}$ The polyaddition of bis(epoxide)s with triazine dichloride proceeded easily to give poly(cyanurate)s with pendant chloromethyl groups. ${ }^{29}$

The polyaddition of bis(epoxide)s with dichlorosilanes using the aforementioned quaternary onium salts also proceeded regioselectively under mild conditions to produce high molecular weight poly(silyl ether)s. ${ }^{30,31}$ The regioselective polyaddition of bis(epoxide)s with phosphonic dichlorides or dichlorophosphates also gave poly(phosphonate)s or poly(phosphate)s with pendant chloromethyl groups. ${ }^{32,33}$ Furthermore, polymers containing both silicon and phosphorus atoms in the polymer backbone were easily synthesized by the polyaddition of bis(epoxide)s with dichlorosilanes and dichlorophosphates. ${ }^{34}$ The polyaddition of bis(epoxide)s with dichlorosilanes was applied to the synthesis of photo-functional polymers silicon-silicon linkages, which acted as negative-type photoresists. ${ }^{35}$ Germanium-containing polymers with pendant chloromethyl groups were also synthesized by polyaddition of bis(epoxide)s with dichlorogermanes, using TBAB as a catalyst. $^{36}$ The polyaddition of bis(epoxide)s with disulfonyl chlorides yielded poly(sulfonate)s with pendant chloromethyl groups. ${ }^{37}$ The polyaddition of bis(epoxide)s with 2,2'-bis[(4chloroformyl)oxyphenyl]propane (BCPP) also proceeded readily to give aliphatic poly(carbonate)s with the chloromethyl groups (Scheme 6). ${ }^{38}$

The interesting characteristics and advantages of these polymer syntheses based on reactions of bis(thiiranes)s, bis(epoxide)s as twofold. (1) Condensation polymers are synthesized by the polyaddition without harmful waste byproducts: this is a synthetic advantage in polymer chemistry and industrial processes from the viewpoint of atom economy, as proposed by Trost. $^{39}$ (2) The polymerization proceeds by forming reactive chloromethyl groups: that is, the pendant chloromethyl groups are introduced simultaneously with the formation of the polymer backbone during the reaction.

The pendant chloromethyl groups are easily converted to other functional groups by conventional reactions, and these reactive polymers are useful starting materials for the synthesis of functional polymers. For example, photo-functional poly(ester)s with norbornadiene moieties in the side chains were prepared by the reaction of the aforementioned reactive poly(ester) with potassium 3-phenyl-2,5-norbornadiene-2-carboxylate using phase transfer catalysts. ${ }^{26} \mathrm{We}$ also successfully synthesized multifunctional photosensitive polymers with posi- 


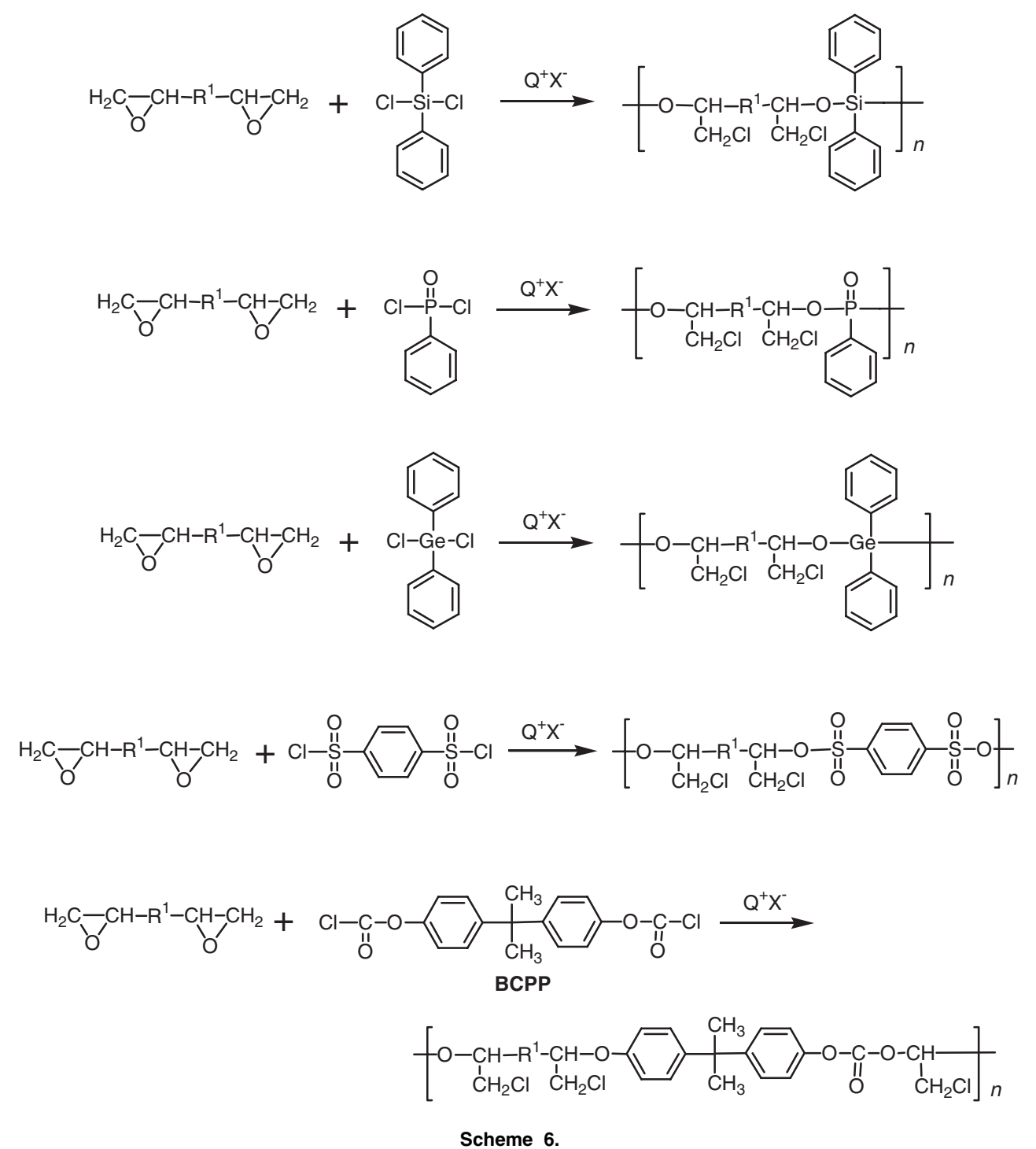

tive-working groups in the main chain and negative-working groups in side chains. ${ }^{40}$ Reactive polyester containing a cyclobutane ring in the main chain and pendant chloromethyl groups was prepared by the polyaddition of bis(epoxide)s with $\beta$-truxiloyl chloride. The reaction of the polyester obtained with (4-dimethylamino)cinnamic acid using 1,8diazabicyclo[5.4.0]-7-undecene (DBU) proceeded with 99\% conversion to provide multifunctional photosensitive polymers (Scheme 7).

Polymerization forming side chains can be applied to synthesis of functional polymers by one-step reactions. The first example is the synthesis of liquid crystalline polymers with kinked aromatic structures in the main chain and biphenylene-type mesogenic groups in side chains (Scheme 8). ${ }^{41,42}$ The polyaddition of bis(epoxide)s with biphenyl $\operatorname{di}(5-(p$-methoxybiphenoxy)-alkanoates $(\mathrm{n}=3-7,10)$ using tetraphenylphosphonium chloride (TPPC) afforded new liquid crystalline polymers, which showed nematic or smectic phases depending on the side chain spacer lengths. ${ }^{42}$

\section{NOVEL SYNTHESIS OF POLYSULFIDES WITH WELL-DEFINED STRUCTURES}

\section{Acyl-transfer Polymerization of Thiiranes}

Polysulfides with controlled molecular weights and narrow molecular weight distributions were prepared by living anionic polymerizations with specific initiators such as potassium carbazyl $^{43}$ or zinc-porphyrin complex. ${ }^{44}$ On the other hand, we designed a novel ring-opening polymerization of cyclic sulfides using carboxylic acid derivatives as initiators and quaternary onium salts as catalysts, in which acyl group transfer occurs in each propagation step.

The polymerization of thiiranes using carboxylic acid derivatives as initiators and quaternary onium salts as catalysts comprises two steps as shown in Scheme 9. ${ }^{45}$ In the initiation step, thiirane monomer reacts rapidly with the initiator to produce a corresponding product (1:1 adduct), $S$-alkylthioester. In the propagation step, the $S$-alkylthioester reacts with 


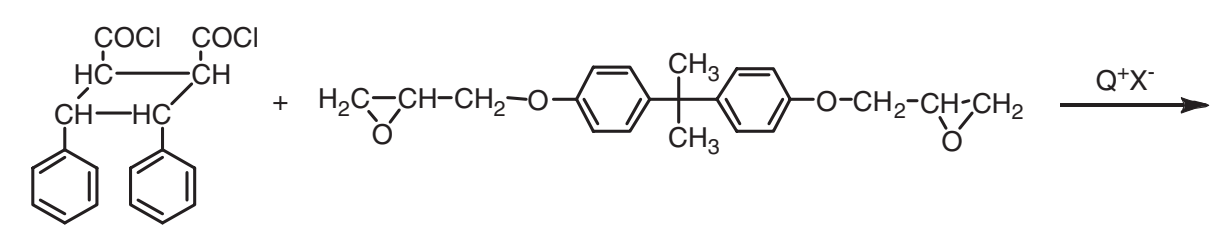

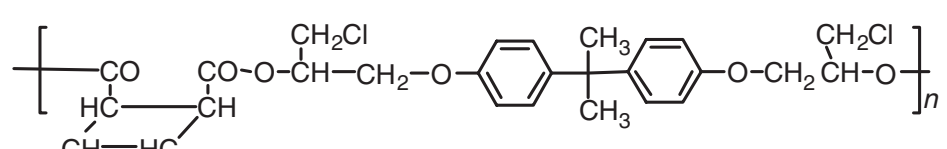<smiles>CC(C)c1ccccc1</smiles><smiles>CC(=O)OC=Cc1ccc(N(C)C)cc1</smiles><smiles>CN(C)c1ccc(C=COCc2ccccc2)cc1</smiles>

Scheme 7.

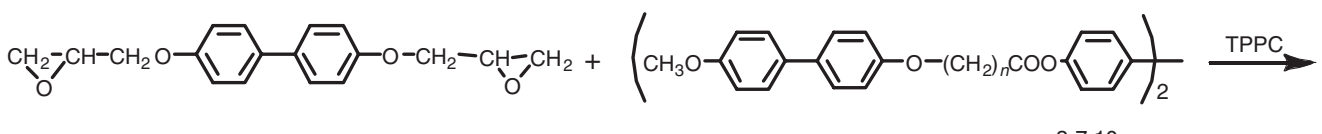

$n=3-7,10$

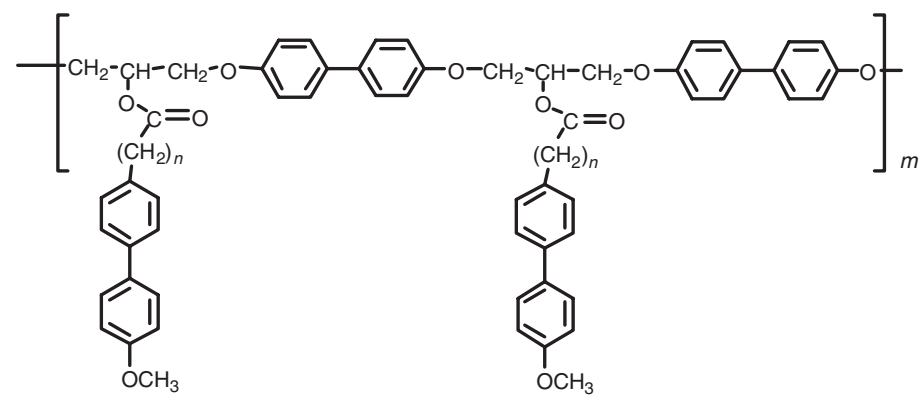

Scheme 8.

monomer to form an addition product, then similar reactions of the $S$-alkylthioester groups of the addition product with monomer proceed in a chain-growth mode.

The polymerization of 3-pheoxypropylene sulfide (PPS) using carboxylic acid derivatives ( $2 \mathrm{~mol} \%$ for PPS) as initiators was carried out in the presence of TBAB or TBAC ( 2 mol \% for PPS) in bulk at $90^{\circ} \mathrm{C}$. The polymerization of PPS with acyl chlorides such as acetyl chloride (AC), dodecanoyl chloride (DC), and BC using TBAC catalyst provided polymers with $M_{\mathrm{n}}$ of about 12000 and a molecular weight distribution $\left(M_{\mathrm{w}} / M_{\mathrm{n}}\right)$ of $c a .1 .3$ in quantitative yields. Furthermore, when the reaction was carried out with $S$-(1-phenoxymethyl-2phenylthio)ethyl thioacetate, which is the 1:1 adduct in the initiation step of the polymerization, the polymer with the same structure as in the polymerization of PPS with PTA was obtained. This means that the polymerization of PPS with PTA is initiated by the selective addition reaction of PTA with PPS, and is followed by chain propagation from PPTA. 


\section{$\underline{\text { Initiation }}$

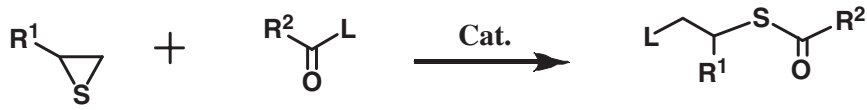 \\ Monomer

\section{$\underline{\text { Propagation }}$}

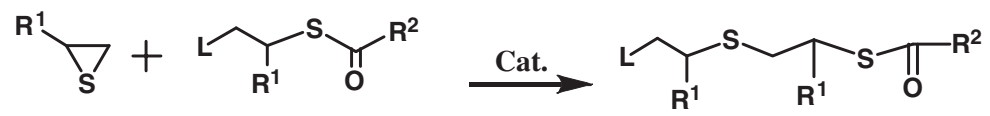

1:1 Adduct

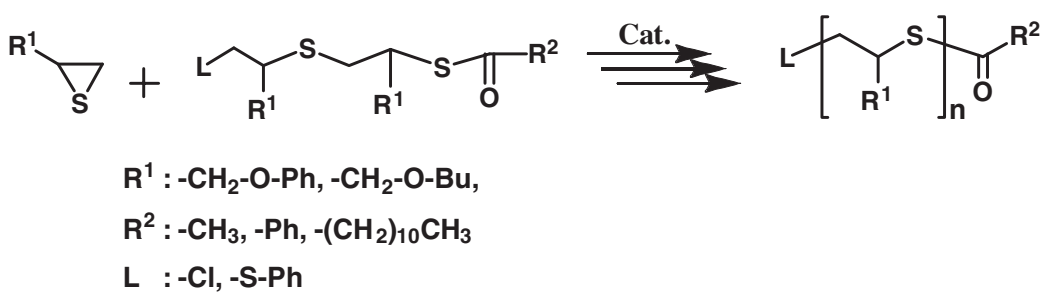

Scheme 9.

The IR spectrum of the polymer obtained from the reaction using PTS showed a weak peak due to $\mathrm{C}=\mathrm{O}$ stretching of the $S$ thioester group at $1691 \mathrm{~cm}^{-1}$. The ${ }^{1} \mathrm{H}$ NMR data indicated that the $\beta$-cleavage of the thiirane ring of PPS occurred selectively. And, a small signal due to $\mathrm{CH}_{3}$ protons of the acetyl group was observed at $2.3 \mathrm{ppm}$. These spectral data proved that the obtained polymer has terminal $S$-acetyl groups, which came from PTA as the initiator. Various thiiranes such as BPS and cyclohexene sulfide (CHS) can be polymerized using PTA as the initiator and $\mathrm{TBAB}$ as the catalyst. ${ }^{46}$ In addition to the thiiranes, four-membered cyclic sulfide, thietane $(\mathrm{TE})^{47}$ was also polymerized in similar reaction systems. The spectral data of the obtained polysulfides proved that the cyclic sulfides were polymerized easily in this reaction system to afford the corresponding polysulfides with terminal $S$-thioester groups. From these results, it is concluded that the polymerization of the cyclic sulfides with carboxylic acid derivatives includes an acyl-group transfer process during each propagating step. The polymerization is named as "Acyl-Transfer Polymerization".

In the polymerization of PPS with PTA, molecular weights increased with the increase of the feed ratios of PPS for PTA. Although this suggests that the polymerization has a characteristic of living polymerization, the molecular weight distribution such as 1.23 , indicates that the molecular weight control is not ideal in this reaction system. We designed initiators with high reactivity such as $S$-(4-chlorophenyl)thio-3,5-dichlorobenzoate (CPTDC) and $S$-phenylthio-(trifluoroacetate) to enhance the initiation process of the polymerization. The polymerization of PPS using CPTDC provided the polymer with narrow molecular weight distribution as $1.13 .^{48}$ In addition, the molecular weight was precisely controlled by the feed ratios of PPS for

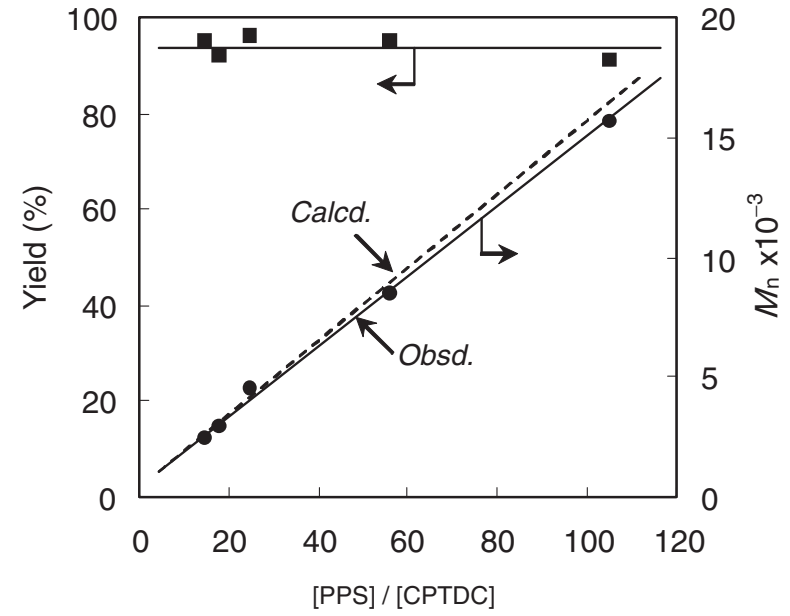

Figure 1. Effect of feed ratios of PPS and CPTDC on the polymerization of PPS (12.5 mmol) using CPTDC as initiator and TBAB as catalyst. Yield [O], $M_{\mathrm{n}}[\mathbf{\square}]$.

CPTDC. The molecular weights were very close to the calculated values as shown in Figure 1. In the polymerization, initiation and propagation proceed smoothly without side reactions such as termination.

\section{Synthesis of Block Copolymers}

Since the polymers obtained from the above reaction system have reactive $S$-acyl groups at the terminals, post-polymerization can be performed using the isolated polymer as a prepolymer. The post-polymerization of PPS using an isolated poly(PPS) with $S$-(3,5-dichlorobenzoyl) groups $\left(M_{\mathrm{n}}=7100\right.$, $\left.M_{\mathrm{w}} / M_{\mathrm{n}}=1.14\right)$ and TBAB in anisole proceeded successfully 
to produce a post polymer with $M_{\mathrm{n}} 10600$ and $M_{\mathrm{w}} / M_{\mathrm{n}} 1.16$ (Scheme 10). This means that the post-polymerization occurred effectively at the $S$-(3,5-dichlorobenzoyl) groups of the polymer ends without side reactions such as termination.

Using the post-polymerization technique, block copolymers of thiiranes and thietane were synthesized (Scheme 11). ${ }^{45}$ AB-type of di-block copolymers, poly(BPS)-block-poly(PPS), poly(BPS)-block-poly(CHS), and poly(BPS)-block-poly(TE) were successfully prepared using the sequential polymerization

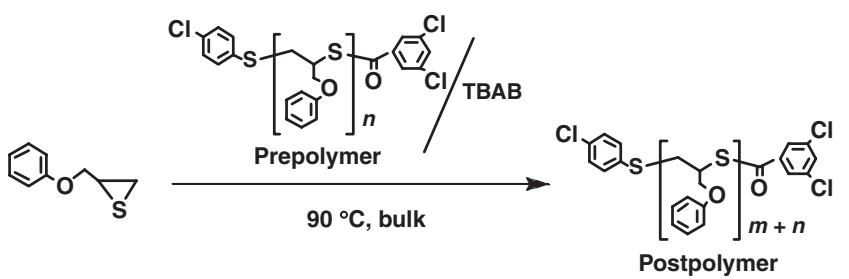

Scheme 10 of the monomers. In addition, ABC-type of tri-block copolymers, poly(BPS)-block-poly(CHS)-block-poly(PPS) and poly(BPS)-block-poly(TE)-block-poly(PPS) were also prepared without side reactions.

\section{Synthesis of Polymers with Complex Architectures}

The ring-opening polymerization of thiiranes was performed using poly-functional initiators. In the case of the polymerization of PPS with 2,2'-bis-[4-(chlorothiophenoxycarbonyl)phenyl]hexafluoropropane (BTPP) and TBAB (Scheme 12), ${ }^{49}$ a center-propagating type polymerization proceeded smoothly to provide the polymer with well-controlled molecular weight and narrow molecular weight distribution as well controlled as in the polymerization of thiiranes with monofunctional initiators.

The synthesis of star-shaped and cyclic poly(sulfide)s was achieved by the ring-opening polymerization of thiiranes with multifunctional initiators derived from calixarenes. Eight-arm star-shaped polysulfides were synthesized by the polymerization of PPS using $p$-t-butylcalix[8]arene (BCA[8]) $S$-thioester

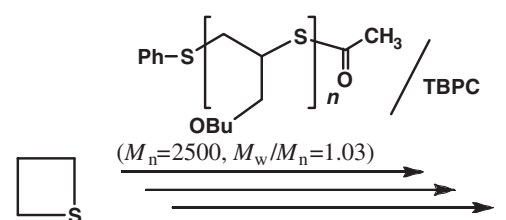

TE

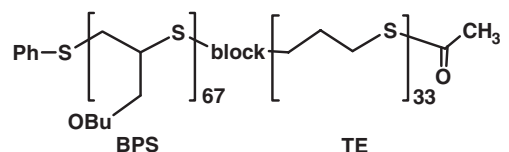

Diblock copolymer $M_{\mathrm{n}}=2800, M_{\mathrm{w}} / M_{\mathrm{n}}=1.13$

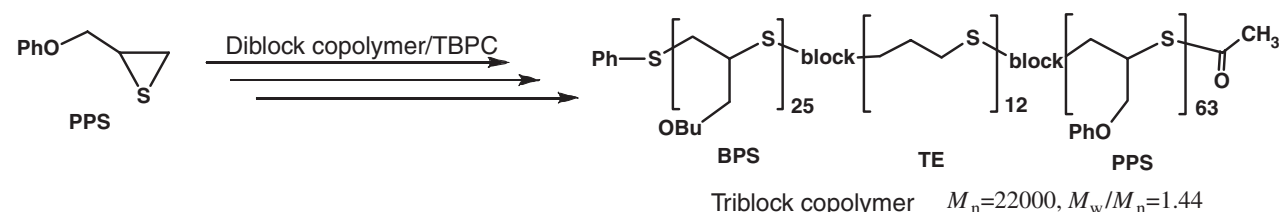

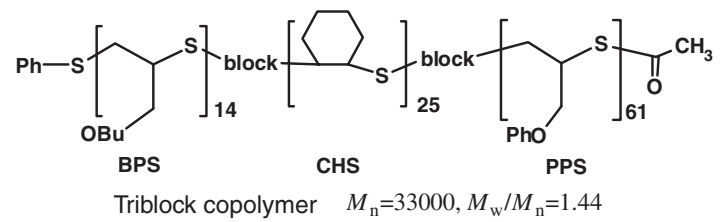

Scheme 11.
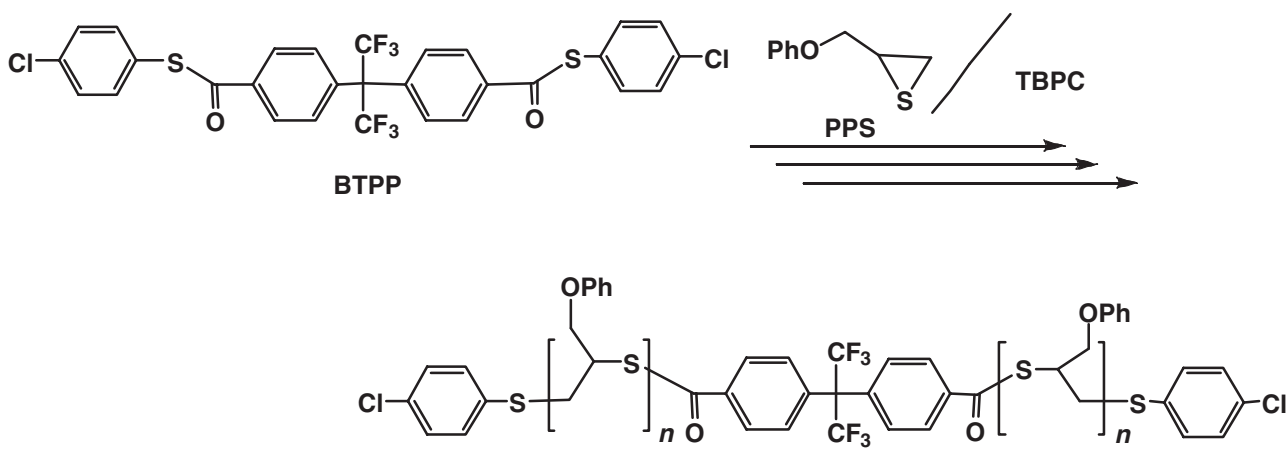

Scheme 12. 


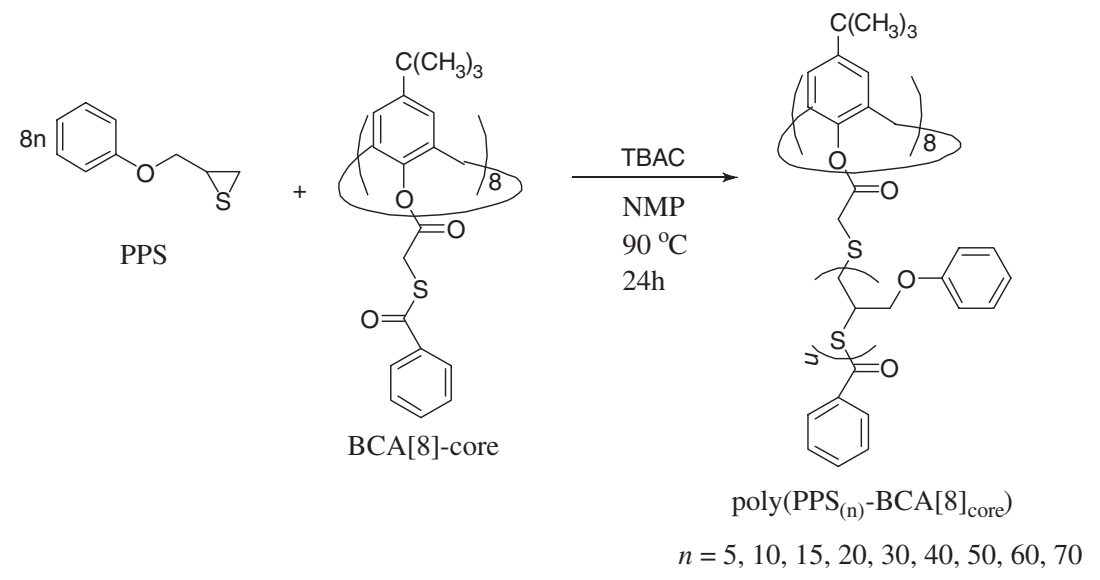

Scheme 13.
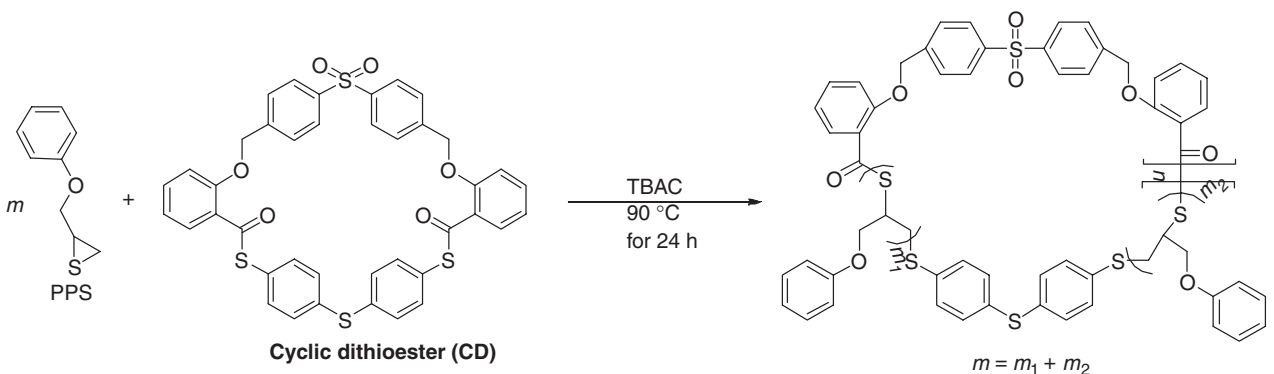

Cyclic polymers

Scheme 14.

derivative as the core initiator and TBAC catalyst (Scheme 13) ${ }^{50}$ The arm-lengths of the star-shaped polysulfides were controlled by feed ratios of $[\mathrm{PPS}] /([$ initiator $] \times 8)$. The refractive-indices index $\left(n_{\mathrm{D}}\right)$ of poly $\left(\mathrm{PPS}_{(\mathrm{n})}-\mathrm{BCA}[8]_{\text {core }}\right) \mathrm{s}$ increased with the sulfur contents. It was found that $n_{\mathrm{D}} \mathrm{S}$ as 1.639 of the star-shaped polymer were higher than those of linear ones $\left(n_{\mathrm{D}}=1.614\right)$ due to their compact structures and consequent high segment density. More recently, Nishikubo reported a similar result that the $n_{\mathrm{D}}$ of star-shaped polystyrene was higher than that of linear polystyrene. ${ }^{51}$

Another interesting study is the synthesis of cyclic polymers of thiiranes using cyclic initiators. The authors found the polymerization of BPS with a macrocyclic $S$-aryl thioester dimmer gave cyclic poly(sulfide)s without production of linear polymers. ${ }^{52} \mathrm{~A}$ continuous insertion reaction of excess PPS into cyclic dithioester (CD) containing two $S$ thioester groups was examined in detail. When the insertion reaction of PPS into $\mathrm{CD}(\mathrm{CD} / \mathrm{PPS}=1 / 50)$ was carried out using TBAC catalyst, the corresponding large cyclic polysulfide with $M_{\mathrm{n}}=42000, \quad M_{\mathrm{w}} / M_{\mathrm{n}}=4.50$ was obtained (Scheme 14). ${ }^{53}$ This is a unique reaction system for the synthesis of cyclic polymers; the size of the cyclic polymers increased with the polymerization as it proceeded. It was also found that an intermolecular ester-exchange reaction occurred as an unexpected reaction during the insertion reaction of PPS into CD. ${ }^{53}$

\section{SYNTHESIS OF NOVEL A-B TYPE SEQUENCE- ORDERED POLYMERS BY COPOLYMERIZATION}

\section{Ring-opening Alternating Copolymerization of Oxiranes with Cyclic $S$-Thioesters}

The most common sequence-ordered polymers are alternating copolymers with an A-B sequence. These polymers have been synthesized by alternating copolymerization of electrondonating olefin monomers with electron-accepting olefin monomers on the basis of charge-transfer complex, ${ }^{54}$ ringopening copolymerization of oxiranes with cyclic carboxylic anhydrides ${ }^{55}$ with carbon dioxide, ${ }^{56}$ and ring-opening copolymerization of oxazolines with lactones. ${ }^{57}$ The alternating terpolymerization of three monomers such as tetrahydrofuran, epichlorohydrin, and phthalic anhydride, ${ }^{58}$ or ethylene phenylphosphonite, acrylonitrile (or methyl acrylate), and carbon dioxide $^{59}$ provided polymers with an A-B-C sequence. On the other hand, in step-growth polymerization system, Ueda developed successful sequence control in the one-step condensation polymerization. ${ }^{60}$

In a series of studies on the reactions of thiiranes or oxiranes with carboxylic acid derivatives, we examined the addition reactions of oxiranes or thiiranes with cyclic $S$-thioesters in the presence of quaternary onium salts or crown ether complexes. The reaction of PGE with $\gamma$-thiobutyrolactone (TBL) using 


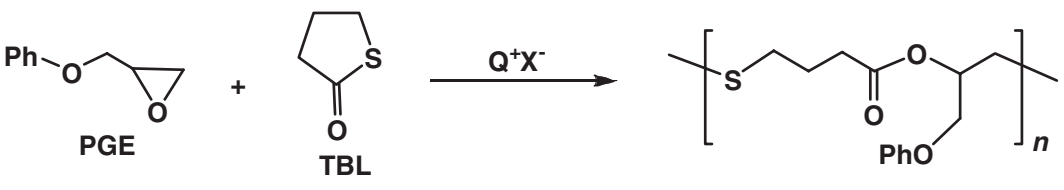

Scheme 15.

\begin{tabular}{|c|c|c|c|c|c|}
\hline \multirow{2}{*}{$\begin{array}{l}\text { Feed ratio } \\
\text { PGE:TBL } \\
100: 0\end{array}$} & \multicolumn{2}{|c|}{$\begin{array}{c}\text { Yield }(\%)^{b)} \\
\text { Exp. [Theory] }\end{array}$} & \multirow{2}{*}{$\begin{array}{c}\text { Composition }{ }^{\mathrm{c})} \\
\text { GPE:TBL } \\
-\end{array}$} & \multirow{2}{*}{$\frac{M_{\mathrm{n}} \times 10^{-4 \mathrm{~d})}}{-}$} & \multirow{2}{*}{$\frac{M_{\mathrm{w}} / M_{\mathrm{n}}{ }^{\mathrm{d})}}{-}$} \\
\hline & 0 & {$\left[\begin{array}{ll}0 & 0\end{array}\right]$} & & & \\
\hline $75: 25$ & 44 & 46 & $50: 50$ & 0.65 & 1.77 \\
\hline $50: 50$ & 93 & 100 & $50: 50$ & 0.95 & 1.99 \\
\hline $25: 75$ & 51 & 55 & $50: 50$ & 0.44 & 1.57 \\
\hline $0: 100$ & 0 & 0 & - & - & - \\
\hline
\end{tabular}

a) The reaction was carried out with PGE and TBL (total $6.0 \mathrm{mmol}$ ) using TBAC $(0.1 \mathrm{mmol})$ at $90^{\circ} \mathrm{C} 1 \mathrm{~h}$ in bulk. b) Insoluble parts in $\mathrm{MeOH}$. c) Determined by ${ }^{1} \mathrm{H}$ NMR. d) Estimated by GPC (THF) based on polystyrene standards.

$3 \mathrm{~mol} \%$ of TBAC without solvent proceeded efficiently at $90{ }^{\circ} \mathrm{C}$ for $1 \mathrm{~h}$ to yield a polymer with $M_{\mathrm{n}} 10000$ and $M_{\mathrm{w}} / M_{\mathrm{n}}$ 1.92. IR, ${ }^{1} \mathrm{H}$ NMR and ${ }^{13} \mathrm{C}$ NMR spectra, GPC profiles and elemental analysis of the resulting polymer demonstrated reasonably that the polymer was an alternating copolymer of PGE with TBL (Scheme 15). ${ }^{61}$ Furthermore, the alternating copolymer was obtained selectively even at various feed ratios of PGE and TBL (Table I) It was quite unexpected that the reaction of TBL gave a copolymer because there are no reports on the ring-opening homopolymerization and copolymerization of TBL.

In addition to PGE, some oxiranes, such as butylglycidyl ether (BGE), 1,2-hexene oxide (HO) and styrene oxide (SO) could be polymerized with TBL to give the corresponding alternating copolymers. In this polymerization, quaternary onium salts with $\mathrm{Cl}^{-}$as the counter anion such as TBAC, showed higher catalytic activity than those, such as TBAB containing $\mathrm{Br}^{-}$.

The alternating copolymerization system exhibited a chaingrowth polymerization character induced by the quaternary onium salts. This conclusion was supported by the results on the effect of feed ratios of monomers and TBAC as shown in Figure 2. Furthermore, an interesting behavior was observed as $M_{\mathrm{n}}$ of the resulting polymer increased with reaction time. This means that the alternating copolymerization system had a stepgrowth polymerization character in the last stage. This was also supported by the following results: when tetrabutylammonium acetate (TBAAc) was used as the catalyst, the increase of the $M_{\mathrm{n}}$ was lower than that of the polymer obtained using TBAC (Figure 3). That is, the increase of the molecular weight of the polymer in the last stage is related to the leaving ability of the counter anions of the quaternary onium salts used. From these results, we concluded the reaction mechanisms for the copolymerization of oxiranes with TBL to be follows (Scheme 16). The initial step is an activation of oxirane by the counter anion of the quaternary onium salt and the activated oxirane reacts

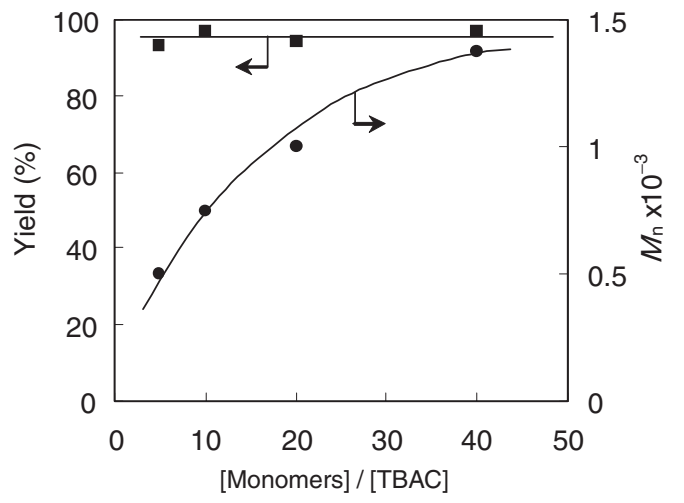

Figure 2. Effect of feed ratios of monomers for TBAC on the copolymerization of PGE (1 mmol) with TBL $(1 \mathrm{mmol})$ using TBAC without solvent at $90^{\circ} \mathrm{C}$ for $2 \mathrm{~h}$. Yield [O], $M_{\mathrm{n}}[\boldsymbol{\square}]$.

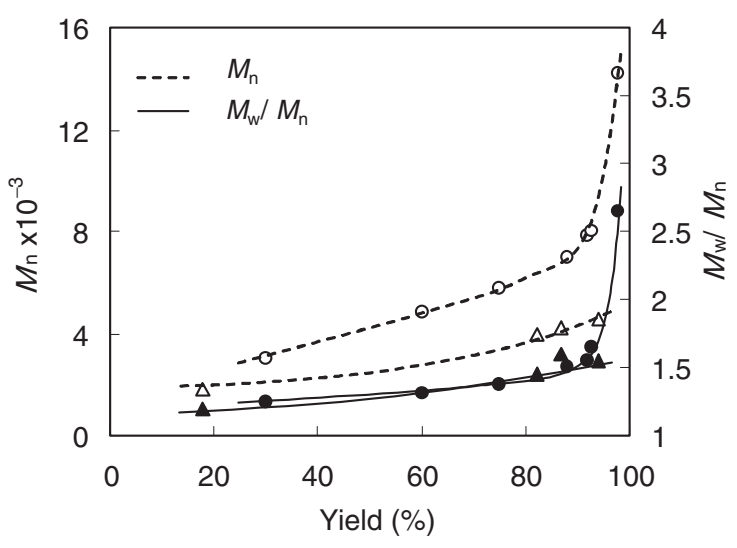

Figure 3. Relatonship between yield and $M_{\mathrm{n}}$ of the polymer on the copolymerization of PGE $(1.0 \mathrm{mmol})$ with TBL $(1.0 \mathrm{mmol})$ using TBAC $(0.03 \mathrm{mmol})$ or TBAAC $(0.03 \mathrm{mmol})$ without solvent at $90^{\circ} \mathrm{C}$. TBAC: $M_{\mathrm{n}}[\bigcirc], M_{\mathrm{w}} / M_{\mathrm{n}}$ [O]. TBAAc: $M_{\mathrm{n}}[\triangle], M_{\mathrm{w}} / M_{\mathrm{n}}[\mathbf{\Delta}]$.

with TBL to produce a thiolate anion. The thiolate anion attacks oxirane to produce activated oxirane. A control experiment confirmed that there was no reaction of TBL with thiolate. The major propagating process in this reaction is repetition of the alternate addition of oxirane and TBL to each propagating species. In addition to the major reaction, coupling reactions of the propagating terminal thiolate anion with active methylene at the initiating terminal of the polymer chains occurred in the late stage.

\section{Ring-opening Alternating Copolymerization of Oxetanes with Cyclic Carboxylic Anhydrides}

We also found the anionic copolymerization of oxetanes with cyclic carboxylic anhydrides to form polyesters using 


\section{Chain growth type reaction}

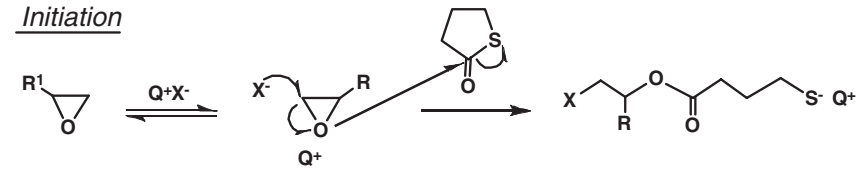

Propagation Step
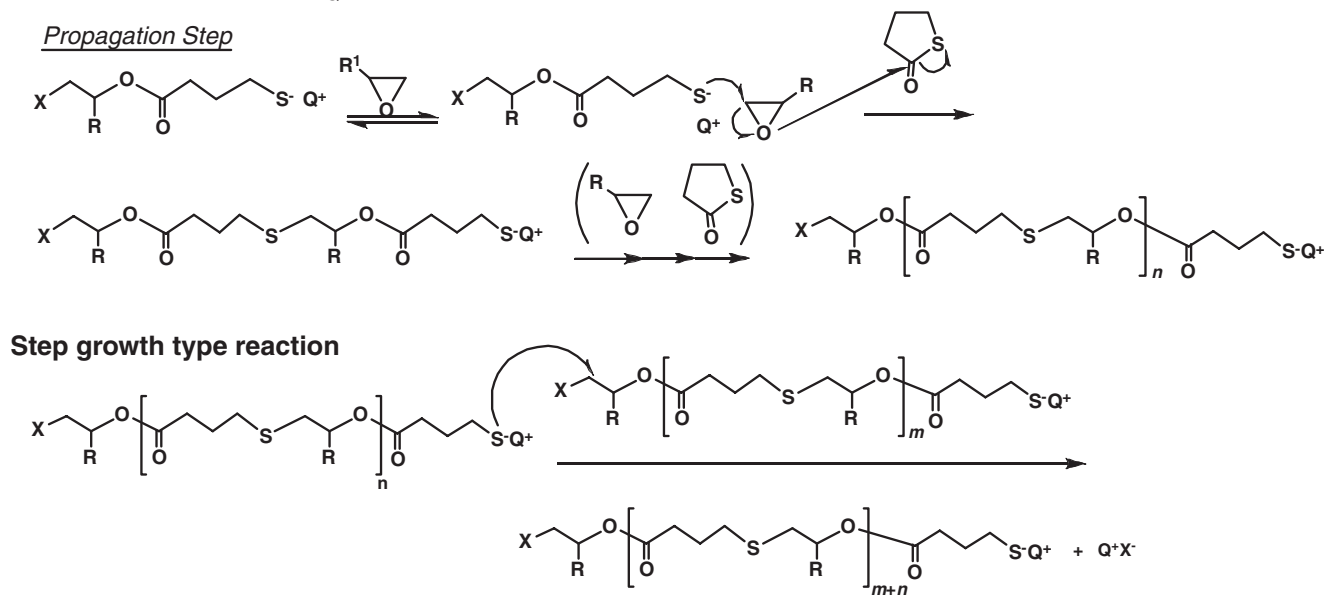

Scheme 16.

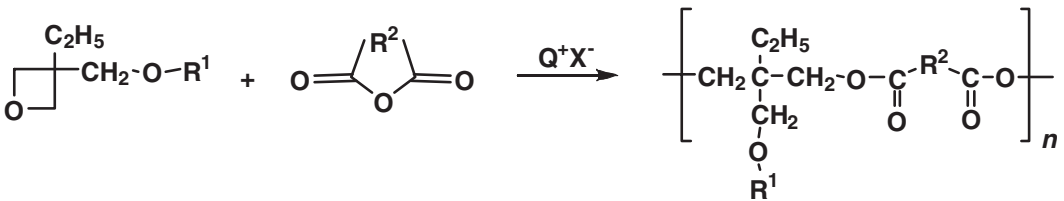

$\mathbf{Q}^{+} \mathbf{X}^{-}$: quaternary onium salts or crown ether complexes

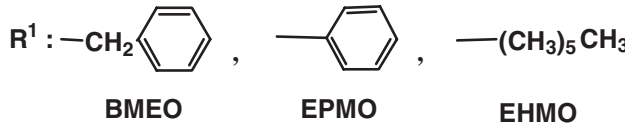

$\mathbf{R}^{2}$ :

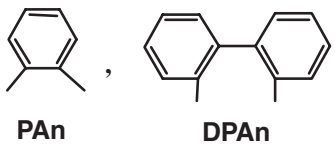

Scheme 17.

quaternary onium salts (Scheme 17). ${ }^{62}$ The reaction of 3 benzyloxymethyl-3-ethyloxetane (BMEO) with phthalic anhydride (PAn) using $5 \mathrm{~mol} \%$ of TBPB without solvents proceeded at $130{ }^{\circ} \mathrm{C}$ for $24 \mathrm{~h}$ to afford an alternating copolymer with $M_{\mathrm{n}} 11000$ and $M_{\mathrm{w}} / M_{\mathrm{n}} 1.28$. The structure of the polymer obtained was clearly identified as an alternating copolymer of BMEO and PAn by IR, ${ }^{1} \mathrm{H}$ NMR, and ${ }^{13} \mathrm{C}$ NMR spectral data. The alternating copolymer was obtained selectively at various feed ratios of BMEO and PAn. The copolymerization of 3ethyl-3-phenoxymethyl oxetane (EPMO) or 3-ethyl-3-hexyl oxetane (EHMO) with PAn also proceeded to provide the corresponding alternating copolymers. The counter anions of the quaternary onium salts play an important role in the initiation process of this copolymerization. The copolymerization appears to be an anionic reaction, although it is well known that oxetanes do not polymerize with anionic catalysts. This is the first synthesis of alternating copolymers of oxetanes with cyclic carboxylic anhydrides. Although the copolymerization of oxetanes with cyclic carboxylic anhydrides using a Lewis acid was reported earlier, the resulting copolymers contained both the alternating copolymer chains of two monomers and polyether chains of the oxetane. ${ }^{63}$

\section{SYNTHESIS OF SEQUENCE-ORDERED POLY- MERS BASED ON THE SELECTIVE TRANSFOR- MATION OF POLYMER BACKBONES}

The sequence control in the alternating copolymerization is essentially based on the difference in reactivity between the used monomers, and the combinations of the monomers 

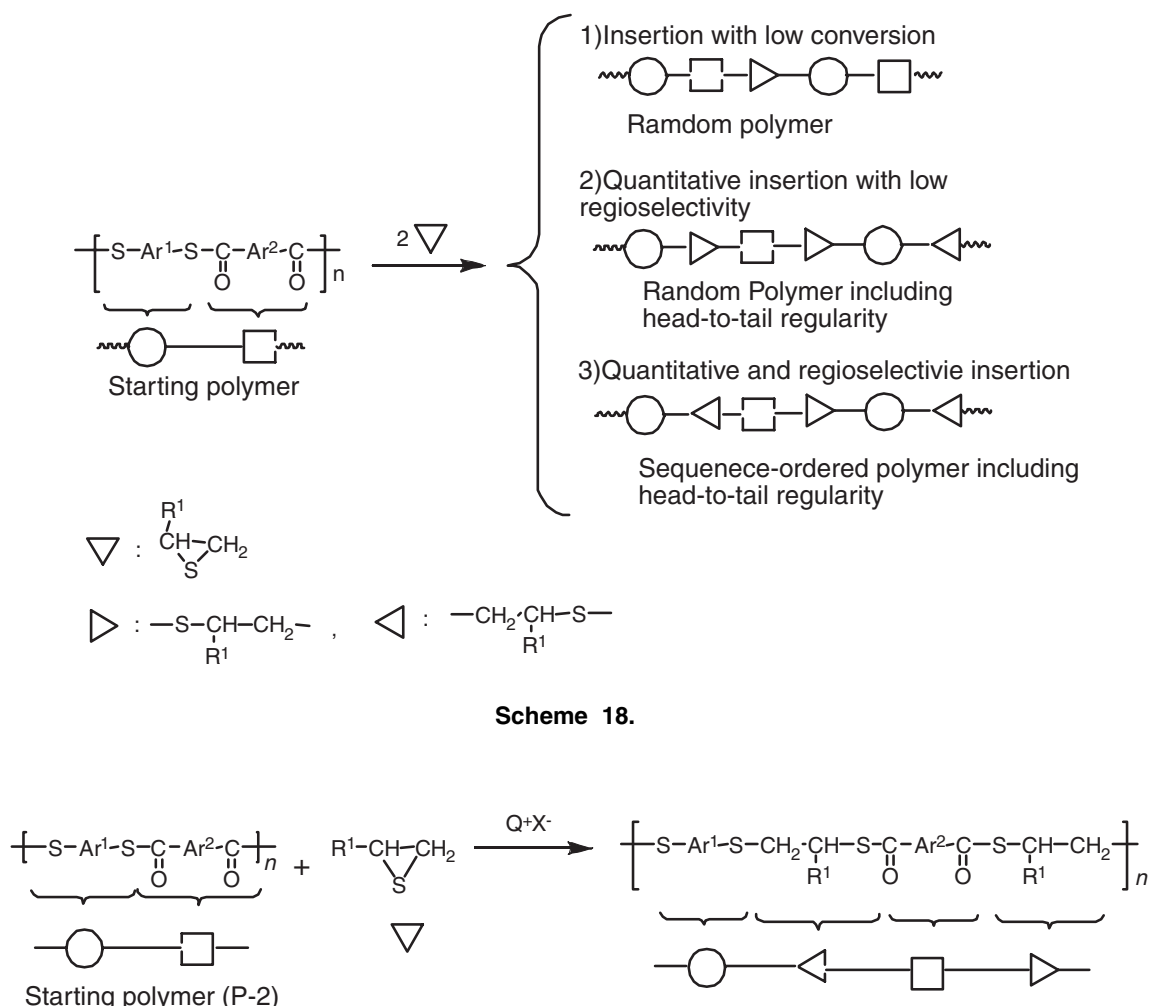

Sequence-Ordered Polymers (P-3)

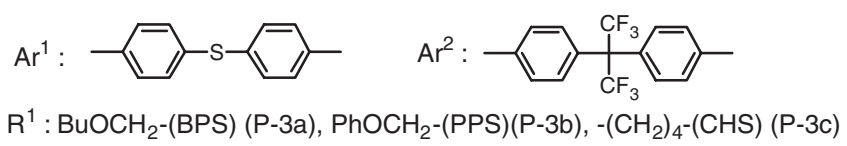

Scheme 19.

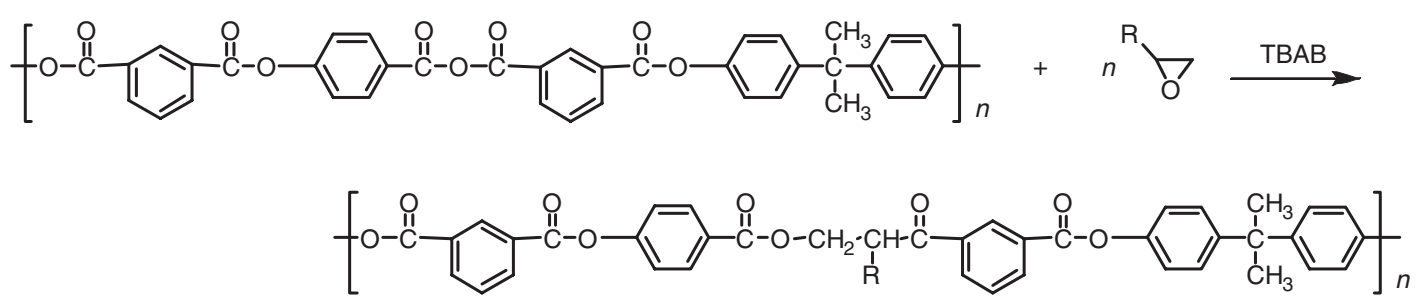

Scheme 20.

applicable to the copolymerization are thus restricted. Furthermore, the synthesis of polymers with a multiple-sequence such as A-B-C-D is still difficult. We have proposed a novel concept for the synthesis of sequence-ordered polymers by the chemoselective and regioselective transformation of the polymer backbone using the insertion reaction of cyclic compounds such as thiiranes and oxiranes. ${ }^{64}$ The concept is presented in Scheme 18. To achieve the sequence-ordered polymers, a selective transformation of the polymer backbone by quantitative insertion is required, because the insertion reaction at low conversion will produce disordered polymers. It is also necessary that the cyclic compound should be inserted with complete regioselectivity (selective ring-opening mode of the oxiranes) to obtain head-to-tail regularity.

The reaction of starting polymer (poly $(S$-aryl thioester)) P-2 $\left(M_{\mathrm{n}}=7800\right)$ with BPS was conducted using $5 \mathrm{~mol} \%$ TBAC (for BPS) in DMAc at $70{ }^{\circ} \mathrm{C}$ for $6 \mathrm{~h}$ to provide a polymer (P-3a) with $96 \%$ yield. ${ }^{65}$ The degree of insertion (DI) of BPS into P-2 was $100 \%$, as estimated by the intensity ratios in the ${ }^{1} \mathrm{H}$ NMR of the resulting polymer. The polymer was thoroughly characterized by the IR, ${ }^{1} \mathrm{H} \mathrm{NMR},{ }^{13} \mathrm{C} \mathrm{NMR}$ spectra, GPC analysis, and elemental analysis. In the ${ }^{13} \mathrm{C}$ NMR spectra of P-2 and P-3a, each signal was assigned to a carbon of the structure $\mathrm{P}-3 \mathrm{a}$ in Scheme 20. In particular, signals due to 

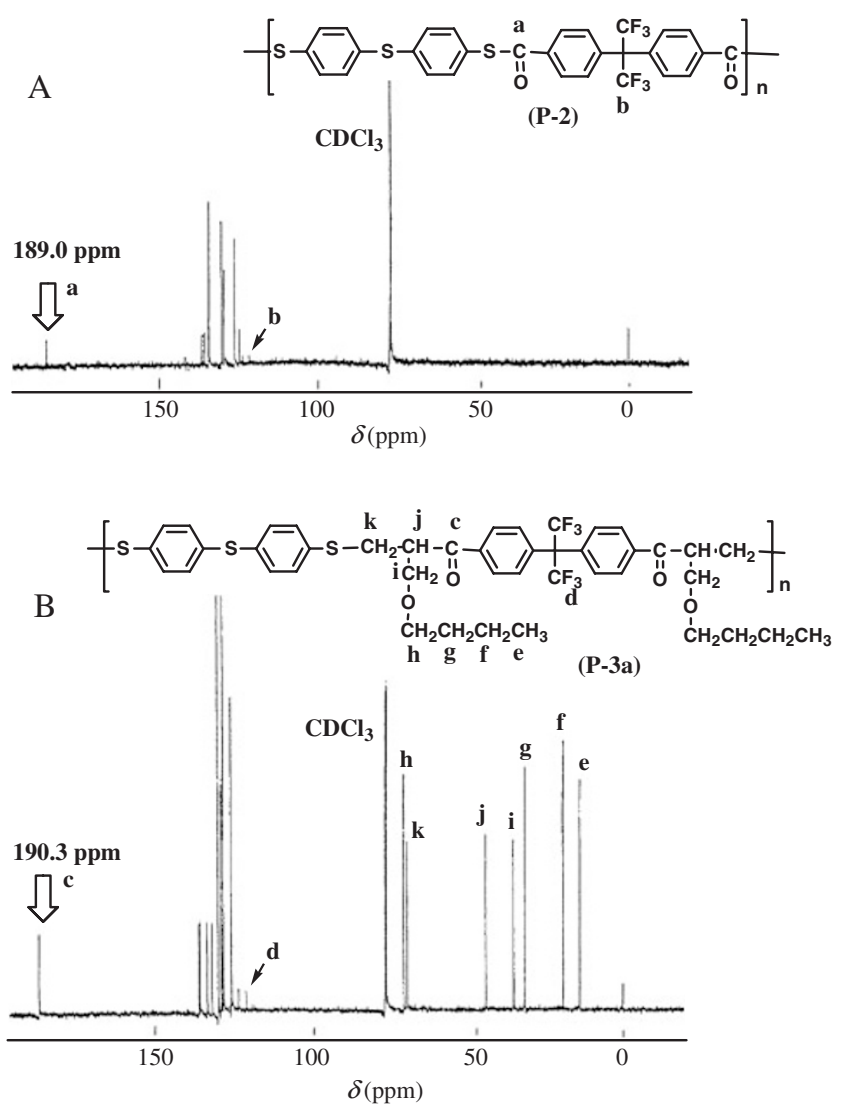

Figure 4. ${ }^{13} \mathrm{C}$ NMR spectra of $\mathrm{P}-2(\mathrm{~A})$ and $\mathrm{P}-3 \mathrm{a}(\mathrm{B})$.

methine and methylene carbons corresponding to the ringopened structure of BPS were observed at 44.34 and 70.06 ppm, respectively, which was supported by the identification of the product from the model reaction. The signal of the $\mathrm{C}=\mathrm{O}$ carbon of the $S$-alkyl thioester group appeared at $190.3 \mathrm{ppm}$, but the signal due to the $\mathrm{C}=\mathrm{O}$ carbon of $\mathrm{P}-2$ at $189.0 \mathrm{ppm}$ was not observed (Figure 4A and 4B).

A unimodal GPC profile of P-3a was observed in a higher molecular weight region compared to that of the starting P-2 $\left(M_{\mathrm{n}}=12000\right)$, and the $M_{\mathrm{n}}$ of P-3a was 13800 . This suggests the insertion of BPS proceeded without any side reactions that induce cleavage of the polymer backbone of poly $(S$-aryl thioester) P-2. All results obtained from the characterizations prove that P-2 is successfully transformed to P-3a with the multiple-sequence represented as $-\bigcirc-\triangleleft-\square-\neg-$ (Scheme 19).

The successful transformation of poly( $S$-thioester) is based on the chemoselectivity and regioselectivity of the reaction of thiiranes with carboxylic ( $S$-thioester)s. The reactivity of carboxylic acid derivatives toward oxiranes in the reaction system using quaternary onium salts, decreases in the order, $S$-aryl ester $>S$-alkyl ester $>O$-aryl ester $>O$-alkyl ester. Therefore, the reaction of thiiranes with $S$-alkylthioester groups in the resulting polymer did not occur during the reaction of thiiranes with poly( $S$-arylthioester). The regioselectivity of the reaction of thiiranes with the poly $(S$-arylthioester)s is supported by the control reaction of thiiranes with carboxylic acid $S$-arylthioester, which is discussed in Section 2.
The reaction of P-2 with BPS proceeded in DMAc at $70^{\circ} \mathrm{C}$ very smoothly with $90 \%$ DI at $1 \mathrm{~h}$, and $100 \%$ in $6 \mathrm{~h}$. It should be noted that the transformation of the polymer backbones proceeds quite smoothly under mild conditions. It is known that the achievement of quantitative polymer reactions is not easy even in reactions of polymer side chains. Various sequenceordered polymers were also obtained based on the transformation of the polymer backbone of P-2 using the insertion of thiiranes such as PPS, CHS. ${ }^{65}$

We also succeeded in the transformation of the polymer backbone of a poly(anhydride) containing ester groups by the insertion of oxiranes using quaternary onium salts as catalysts (Scheme 20). ${ }^{66}$ In the reaction, insertion of oxiranes into the anhydride groups occurred chemoselectively with $100 \%$ conversion to give aliphatic poly(ester)s, without the reaction of oxiranes with carboxylic acid aryl ester in the polymer. In this case, the sequences including head-to head or head-to tail regularity of the resulting poly(ester)s, that is, the ring-opening mode of the oxiranes on the insertion could not be evaluated because of complicated NMR signals. It was demonstrated that the transformation of polymer backbones by insertion of cyclic compounds such as thiiranes and oxiranes is a useful synthetic method for sequence-ordered polymers with multiple-sequences.

\section{CONCLUSION}

We have described here syntheses of polymers with welldefined structures on the basis of novel regioselective addition reactions of thiiranes with certain reagents under neutral conditions. The regioselective reactions of the cyclic compounds with active chlorides were successfully applied to novel polymerization forming pendant chloromethyl groups. The polymerization afforded novel reactive polymers, such as poly(ester)s, poly( $S$-thioester)s, poly(ether)s, poly(cyanurate)s, poly(silyl ether)s, poly(phosphonate)s, and poly(sulfonate)s. The synthesized reactive polymers can be used as starting materials for the synthesis of various functional polymers. Poly(sulfide)s with controlled molecular weight and molecular weight distribution were synthesized by Acyl-Transfer Polymerization of thiiranes with carboxylic acid $S$-thioesters, in which acyl-groups transfer at the propagating termini of growing chains. This polymerization system affords a useful synthetic method for the controlled macromolecular architecture of block copolymers and polymers with complex architectures such as star-shaped polymers and cyclic polymers. Novel alternating copolymerizations of oxiranes with cyclic $S$ thioesters, and oxetanes with cyclic carboxylic anhydrides were also developed. Interestingly, the copolymerizations combined chain-growth and step-growth polymerization. Some sequenceordered polymers were synthesized by the selective transformation of polymer backbones of condensation polymers using the insertion of thiiranes or oxiranes. Thus, the synthesis of polymers with well-defined structures can be achieved by simple synthetic methodology based on new selective addition reactions of thiiranes and cyclic ethers. 
Acknowledgment. This work was financially supported by a Grant-in-Aid for Science Research from the Ministry of Education, Science, and Culture of Japan, which is gratefully acknowledged.

\section{Nomenclature}

\section{Compounds}

Acetyl chloride

Adipoyl chloride

Benzoyl chloride

3-Benzyloxymethyl-3-ethyloxetane

$S$-Benzyl thioacetate

2,2'-bis[(4-chloroformyl)oxyphenyl]propane

Bisphenol A di(2,3-epithiopropyl)ether

Butandiol di (2,3-epithiopropyl)ether

3-Butoxypropylene sulfide

Butylglycidyl ether

$p$ - $t$-butylcalix[8]arene

$S$-(4-chlorophenyl)thio-3,5-dichlorobenzoate

Cyclic dithioester

Cyclohexene sulfide

1,8-Diazabicyclo[5.4.0]-7-undecene

Diphenic anhydride

Dodecanoyl chloride

3-Ethyl-3-hexyl oxetane

3-Ethyl-3-phenoxymethyl oxetane

1,2-Hexene oxide

3-Phenoxypropyrenesulfide

$S$-Phenyl thioacetate

3-Pheroxypropylene sulfide

Phthalic anhydride

Quaternary onium halides

Sebacoyl chloride

Styrene oxide

Terephthaloyl chloride

Tetrabutylammonium acetate

Tetrabutylammonium bromide

Tetrabutylammonium chloride

Tetrabutylammonium iodide

Tetrabutylphosphonium bromide

Tetraphenylphosphonium chloride

Thietane

$\boldsymbol{\gamma}$-Thiobutyrolactone

Abbreviations
AC
APC
BC
BMEO
BZTA
BCPP
BPTE
BDTE
BPS
BGE
BCA[8]
CPTDC
CD
CHS
DBU
DPAn
DC
EHMO
EPMO
HO
PPS
PTA
PPS
PAn
Q ${ }^{-}$
SCC
SO
TPC
TBAAc
TBAB
TBAC
TBAI
TBPB
TPPC
TE
TBL

Received: April 17, 2008

Accepted: June 22, 2008 Published: August 12, 2008

\section{REFERENCES}

1. T. Nishikubo and A. Kameyama, "Synthesis and Reaction of Polymers," S. Inoue, Ed., Kyoritsu Shupan, Tokyo, 1996, pp. 4-119.

2. "Epoxy Resin Chemistry," R. S. Bauer, American Chemical Society, Washington D.C., 1979.

3. Z. Jedliński, J. Kasperczyk, A. Dworak, and B. Matuszewska,
Makromol. Chem., 183, 587 (1982).

4. "Aziridines and Epoxides in Organic Synthesis," L. C. Nielson, E. N. Jacobsen, and A. K. Yudin, Ed., Wiley-VCH, Weinheim, 2006, pp. 229-269.

5. T. Nishikubo and A. Kameyama, Prog. Polym. Sci., 18, 963 (1993).

6. E. J. Goethals, Adv. Polym. Sci., 23, 103 (1977).

7. G. K. Helmkamp, D. J. Pettitt, J. R. Lowell, W. R. Mabey, and R. Wolcot, J. Am. Chem. Soc., 88, 1030 (1966).

8. Y. Taguchi, K. Yanagiya, I. Shibuya, and Y. Suhara, Bull. Chem. Soc. Jpn., 60, 727 (1987).

9. M. Furukawa, K. Nagata, and Y. Kojima, Chem. Pharm. Bull., 20, 2262 (1972).

10. Y. Taguchi, I. Shibuya, and Y. Suhara, Nippon Kagakukaishi, 1, 63 (1989).

11. T. Matsuda, K. Yamazaki, H. Ide, K. Noda, and K. Yamagata, Jpn. Patent 49013164 (1974).

12. S. Oae, "Organic Sulfur Chemistry: Structure and Mechanism," CRC Press, Boca Raton, 1991.

13. S. W. Benson, Chem. Rev., 78, 23 (1978).

14. M. Yus and F. Foubel, "Reviews on Heteroatom Chemistry," S. Oae, Ed., MYU, Tokyo, 1997, pp. 73-107.

15. P. Sigwalt and N. Spassky, "Ring-opening Polymerization," K. J. Ivin and T. Saegusa, Ed., Elsevier Applied Science Publishers, Essex, 1984, pp. 603-714.

16. E. J. Goethals and J. M. Greeraert, Plast. Eng., 40, 223 (1997).

17. E. Kameyama, M. Nakajima, and T. Kuwamura, Bull. Chem. Soc. Jpn., 45, 3222 (1972).

18. K. Funahashi, Chem. Lett., 1043 (1978).

19. N. Iranpoor, H. Firouzabadi, and A. A. Jafari, Synth. Commun., 33, 2321 (2003).

20. A. Kameyama, M. Kiyota, and T. Nishikubo, Tetrahedron Lett., 35, 4571 (1994).

21. S. Sandler and W. Karo, "Polymer Synthesis," Academic Press N.Y., 1996, pp. 62-67.

22. B. Jerman and D. Fles, J. Polym. Sci., Part A: Polym. Chem., 14, 1117 (1976).

23. C. G. Overberger and J. K. Weise, J. Am. Chem. Soc., 90, 3533 (1968).

24. C. G. Overberger and J. K. Weise, J. Am. Chem. Soc., 90, 3525 (1968).

25. A. Kameyama, Y. Kimura, and T. Nishikubo, Macromolecules, 30, 6494 (1997).

26. A. Kameyama, S. Watanabe, E. Kobayashi, and T. Nishikubo, Macromolecules, 25, 2307 (1992).

27. A. Kameyama and T. Nishikubo, React. Funct. Polym., 30, 235 (1996).

28. T. Nishikubo, A. Kameyama, and K. Ogawa, J. Polym. Sci., Part A: Polym. Chem., 35, 3791 (1997).

29. T. Nishikubo, A. Kameyama, and T. Yaguchi, J. Polym. Sci., Part A: Polym. Chem., 38, 4006 (2000).

30. T. Nishikubo, A. Kameyama, and N. Hayashi, Polym. J., 25, 1003 (1993).

31. T. Nishikubo, A. Kameyama, Y. Kimura, and K. Fukuyo, Macromolecules, 28, 4361 (1995).

32. T. Nishikubo, A. Kameyama, and S. Minegishi, Macromolecules, 27, 2641 (1994).

33. T. Nishikubo, A. Kameyama, and S. Minegishi, Macromolecules, 28 , 4810 (1995).

34. S. Minegishi, A. Kameyama, and T. Nishikubo, React. Funct. Polym., 30, 317 (1996).

35. A. Kameyama, N. Hayashi, and T. Nishikubo, "Polymeric Materials for Microelectronic Application" (ACS Symposium Series 579), S. Tagawa, K. Horie, and H. Ito, Ed., Am. Chem. Soc., Washington D.C., 1994, pp. 443-453.

36. T. Nishikubo, A. Kameyama, and Y. Kimura, Polym. J., 27, 934 (1995).

37. S. Minegishi, S. Kameyama, and T. Nishikubo, J. Polym. Sci., Part A: 
Polym. Chem., 36, 249 (1998).

38. T. Yashiro, K. Matsushima, A. Kameyama, and T. Nishikubo, Macromolecules, 33, 7737 (2000).

39. B. M. Trost, Science, 254, 1471 (1991).

40. T. Nishikubo, A. Kameyama, N. Hayashi, and R. Nakagome, High Perform. Polym., 7, 245 (1995).

41. B. Q. Chen, A. Kameyama, and T. Nishikubo, Macromolecules, 32, 6485 (1999).

42. B. Q. Chen, A. Kameyama, and T. Nishikubo, J. Polym. Sci., Part A: Polym. Chem., 38, 988 (2000).

43. A. Boileau and P. Sigwalt, Bull Chim. France, 1418 (1968).

44. T. Aida, K. Kawaguchi, and S. Inoue, Macromolecules, 23, 3887 (1990).

45. A. Kameyama, K. Shimotsuma, and T. Nishikubo, Macromol. Rapid Commun., 15, 335 (1994).

46. A. Kameyama, K. Shimotsuma, and T. Nishikubo, Polym. J., 28, 68 (1996).

47. A. Kameyama, K. Shimotsuma, H. Kawashima, and T. Nishikubo, presented at the 5th SPSJ International Polymer Conference, Senri Life Science Center, Senri, Osaka, Japan, November 28-December 2, 1994.

48. S. Kawakami, A. Kameyama, and T. Nishikubo, Polym. Prepr. Jpn., 44, 202 (1995)

49. S. Kawakami, A. Kameyama, M. Miyata, and T. Nishikubo, Polym. J., 29, 550 (1997).

50. H. Kudo, H. Inoue, T. Nishikubo, and T. Anada, Polym. J., 38, 289 (2006).
51. H. Kudo, H. Aoki, and T. Nishikubo, Chem. Lett., 37, 282 (2008).

52. T. Yashiro, A. Kameyama, and T. Nishikubo, Polym. Prepr. Jpn., 47, 210 (1998).

53. H. Kudo, S. Makino, A. Kameyama, and T. Nishikubo, Macromolecules, 38, 5964 (2005).

54. G. Ordian, "Principle of Polymerization," 3rd ed., John Wiley \& Sons, New York, 1991, pp. 453-531.

55. J. Luston and F. Vass, Adv. Polym. Sci., 56, 91 (1984).

56. S. Inoue, Prog. Polym. Sci. Jpn., 8, 1 (1975).

57. T. Saegusa, H. Ikeda, and H. Fujii, Macromolecules, 5, 354 (1972).

58. H. L. Hsieh, Polym. Prepr. (Am. Chem. Soc. Div. Polym. Chem.), 12 157 (1972)

59. T. Saegusa, S. Kobayashi, and Y. Kimura, Macromolecules, 10, 68 (1977).

60. M. Ueda, Prog. Polym. Sci., 24, 699 (1999).

61. T. Nishikubo, A. Kameyama, and S. Kawakami, Macromolecules, 31, 4746 (1998).

62. A. Kameyama, K. Ueda, H. Kudo, and T. Nishikubo, Macromolecules, 35, 3792 (2002).

63. D. Takeuchi, T. Aida, and T. Endo, Macromol. Rapid Commun., 20, 646 (1999).

64. A. Kameyama, Y. Murakami, and T. Nishikubo, Macromolecules, 29, 6676 (1996).

65. A. Kameyama, Y. Murakami, and T. Nishikubo, Macromolecules, 32, 1407 (1999).

66. T. Yashiro, A. Kameyama, T. Nishikubo, and H. R. Kricheldorf, Polym. J., 31, 102 (1999).

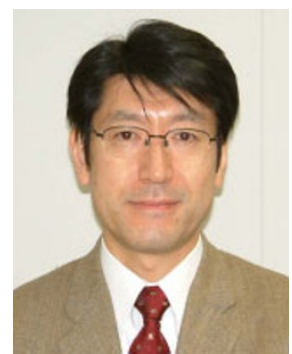

Atsushi Kameyama was born in Miyagi, Japan, in January 9th, 1959. He received his Doctorate Degree from Tokyo Institute of Technology, Japan in 1990. He did postdoctoral research at Cornell University, USA, with Professor Christopher K. Ober (1995-1996). His career at Kanagawa University has included being Research Associate (1990), Associate Professor (2000), and Full Professor (2006). He has published about 140 papers in refereed scientific journals and 27 books and reviews. He has made an invited lecture at international scientific conferences. His research interest covers synthesis and self-organization of polymers with well-defined structures, preparation of microspheres, and novel photopolymerization system.

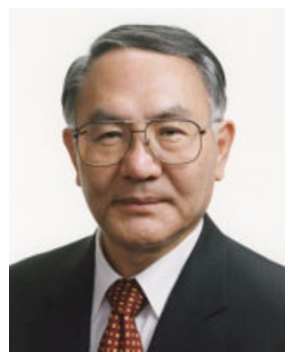

Tadatomi Nishikubo received Bachelor degree in Applied Chemistry in Kanagawa University, Japan, in 1967. He joined NOK. Co., Ltd., Japan in 1967-1978. He received Doctorate degree in Electronic Chemistry from Tokyo Institute of Technology, Japan, in 1976. His career at Kanagawa University has included being Lecturer (1978), Associate Professor (1980), Full Professor (1986), Dean (2000-2005) and Vice-President (2006-2007). He worked with Dr. R. W. Lenz as a visiting faculty at the University of Massachusetts, USA, in 1981-1982. Since 2006, he is Trustee of Kanagawa University. He has been the authors of over 300 publications, including original research papers, reviews, and books. He has made 25 plenary and invited lectures at international scientific conferences. His research interest covers synthesis and reactions of new photo-functional polymers and synthesis of macromolecules with well-defined topological structures. 